\title{
Validation of a food frequency questionnaire to assess habitual dietary vitamin D intake using the method of triads
}

\author{
Á. Hennessy and M. Kiely \\ School of Food and Nutritional Sciences, University College Cork, Republic of Ireland
}

In most food frequency questionnaire (FFQ) validation studies, the questionnaire data are compared with a reference method (food record, 24-hr recalls, diet history) in order to estimate its correlation with the subject's true habitual intake. The availability of a biochemical measure of nutrient status allows for a triangular comparison between the FFQ, the reference method and the biomarker, and for quantitative estimate of the questionnaire's validity coefficient ${ }^{(1)}$. The aim of this study was to assess the validity of a FFQ designed to assess habitual vitamin D intake using the method of triads. Using national food consumption data ${ }^{(2)}$, the contribution of foods to vitamin D intake in the population were quantified using the population proportion method ${ }^{(3)}$. The foods which represent 95 $\%$ of the food sources of vitamin D intake in the population were identified ${ }^{(2)}$ and a list of questions to assess habitual intake of these foods (frequency and portion size ${ }^{(4)}$ ) were developed. The FFQ was administered in 120 subjects and a 14-day diet history (DH) was used as the "reference" dietary assessment method. Serum 25-hydroxyvitamin D concentrations (s25OHD) were quantified. Pearson correlation coefficients were calculated between each of the assessment methods and validity coefficients were calculated using the method of triads ${ }^{(1)}$. The $95 \%$ confidence intervals (CIs) for the validity coefficients were estimated using bootstrap sampling. All statistical analyses were performed in SPSS Version 21 (IBM SPSS).

Pearson correlation coefficients between each of the assessment methods of habitual vitamin D intake and validity coefficients as calculated by the method of triads in 120 subjects.

Correlation coefficients

\begin{tabular}{|c|c|c|}
\hline $\begin{array}{l}r_{Q R} \\
0.712(0.584,0.840)\end{array}$ & $\begin{array}{l}r_{Q B} \\
0.243(0.063,0.421) \\
\text { Validity coefficients }\end{array}$ & $\begin{array}{l}r_{R B} \\
0.269(0.091,0.450)\end{array}$ \\
\hline $\begin{array}{l}\rho_{Q T} \\
0.800(0.636,0.886)\end{array}$ & $\begin{array}{l}\rho_{R T} \\
0.886(0.919,0.948) \\
\text { Range for the validity coefficient }{ }^{\mathrm{a}}\end{array}$ & $\begin{array}{l}\rho_{B T} \\
0.304(0.099,0.475)\end{array}$ \\
\hline $\begin{array}{l}F F Q \\
0 \cdot 243-0 \cdot 800\end{array}$ & $\begin{array}{l}\text { DH } \\
0 \cdot 269-0 \cdot 886\end{array}$ & $\begin{array}{l}s 25 O H D \\
0 \cdot 243-0 \cdot 304\end{array}$ \\
\hline
\end{tabular}

$Q=$ food frequency questionnaire, $R=$ diet history, $B=$ biomarker (i.e. s25OHD), $T=$ true value

$r_{Q B}$, correlation between FFQ and s25OHD; $r_{Q R}$, correlation between FFQ and diet history; $r_{R B}$, correlation between diet history and s $25 \mathrm{OHD} ; \rho Q T$, validity

coefficient of the FFQ; $\rho R T$, validity coefficient of the diet history; $\rho B T$, validity coefficient of the biomarker.

${ }^{\text {a }}$ The lower limit is $r_{Q B}$ for the FFQ and s25OHD and $r_{R B}$ for the diet history, and the upper limit is calculated by the method of triads.

The correlation between estimates of vitamin D intake by the FFQ and the diet history was high, while the correlations between estimates of vitamin D intake with s25OHD concentrations were similar between the two dietary assessment methods. The overall validity coefficient of the FFQ calculated using the method of triads was 0.800 (95\% CI: $0.636,0.886)$, indicating high validity. The FFQ developed in this study is suitable for estimating habitual intake of vitamin D.

1. Kaaks RJ (1997) Am J Clin Nutr 65, 1232S-1239S.

2. Irish Universities Nutrition Alliance (2001) Dublin: Food Safety Promotion Board.

3. Krebs-Smith S, Kott P, Guenther P (1989) J Am Diet Assoc 89, 671-676.

4. Nelson M, Atkinson M, Meyer J (1997) A photographic atlas of food portion sizes. England: Food Standards Agency. 